\title{
SPECTRAL MAPPING THEOREMS ON A TENSOR PRODUCT
}

\author{
BY ROBIN HARTE
}

Communicated by Robert G. Bartle, August 17, 1972

1. Introduction. By computing the joint spectrum [5], [6] for certain systems of elements in a tensor product [3], [11] of Banach algebras, and applying the spectral mapping theorem in several variables [5], [6], [7], we find that we can determine the spectrum of certain linear operators, notably the tensor product $S \otimes T$ discussed by Brown and Pearcy [1], $[\mathbf{1 2}]$. We can also see that the spectrum of an "operator matrix" [4], [10] is what it ought to be, and recover the results of Lumer and Rosenblum [10] about the multiplication operators $L_{S} R_{T}$ and $L_{S}+R_{T}$. Full proofs, and more detail, will appear elsewhere $[8]$.

2. Left and right spectra. Suppose that $A$ is a complex Banach algebra, with identity 1 . Then the joint spectrum of a system of elements $a \in A^{n}$ is the union of the left spectrum and the right spectrum [5, Definition 1.1]:

$$
\sigma_{A}^{\text {joint }}(a)=\sigma_{A}^{\text {left }}(a) \cup \sigma_{A}^{\text {right }}(a)
$$

where

$$
\sigma_{A}^{\text {left }}(a)=\left\{s \in C^{n}: 1 \notin \sum_{j=1}^{n} A\left(a_{j}-s_{j}\right)\right\}
$$

and

$$
\sigma_{A}^{\mathrm{right}}(a)=\left\{s \in C^{n}: 1 \notin \sum_{j=1}^{n}\left(a_{j}-s_{j}\right) A\right\} .
$$

The spectral mapping theorem [5, Theorem 3.2] is the equality

$$
\sigma_{A}^{\text {joint }} f(a)=f \sigma_{A}^{\text {joint }}(a)
$$

valid for a commuting system of elements $a \in A^{n}$ and a system $f=\left(f_{1}, f_{2}, \ldots, f_{m}\right)$ of polynomials in $n$ complex variables. Equality (2.4) is also valid for left and right spectra separately; it extends [7, Theorem 4.2] to certain noncommuting systems of elements, where of course the idea of a "polynomial" has to be extended. Here we take a "polynomial in $n$ variables" to be an element of the free complex algebra-with-identity Poly $_{n}$ on $n$ generators $z_{j}$; for an arbitrary system of elements $a \in A^{n}$, the mapping $f \rightarrow f(a):$ Poly $_{n} \rightarrow A$ is a homomorphism which preserves

AMS (MOS) subject classifications (1970). Primary 47D99, 46H99; Secondary 47A10, 46M05.

Key words and phrases. Joint spectrum, spectral mapping theorem, tensor product, uniform crossnorm, operator matrix, multiplication operator. 
identity and sends each $z_{j}$ into the corresponding $a_{j}$, and then a system $f=\left(f_{1}, f_{2}, \ldots, f_{m}\right) \in$ Poly $_{n}^{m}$ defines a mapping $f: A^{n} \rightarrow A^{m}$.

It will be convenient, for what follows, if we summarize the spectral mapping theorems for a composite system of elements $(a, b) \in A^{n+m}$ associated with two systems $a \in A^{n}$ and $b \in A^{m}$. It is also convenient here to work explicitly with the left spectrum (2.2): The arguments for the right spectrum are obviously exactly similar, and can be obtained formally by "reversing products" in the algebra $A$; then we obtain usually the corresponding statement for the joint spectrum by taking unions.

As a convenient abbreviation, write [7, Definition 1.1]

$$
\sigma_{a=s}^{\text {left }}(b)=\left\{t \in \sigma^{\text {left }}(b):(s, t) \in \sigma^{\text {left }}(a, b)\right\},
$$

for arbitrary systems of elements $a \in A^{n}, b \in A^{m}$ and scalars $s \in C^{n}$. Also

$$
\sigma_{a=a}^{\text {left }}(b)=\bigcup\left\{\sigma_{a=s}^{\text {left }}(b): s \in \sigma^{\text {left }}(a)\right\} .
$$

Lemma 1 [7, Theorem 2.3]. If $a \in A^{n}, b \in A^{m}, s \in C^{n}$ and $f \in$ Poly $_{n+m}^{p}$, and if each $a_{j}$ commutes with each $b_{k}$, then there is equality

$$
\sigma_{a=s}^{\text {left }} f(a, b)=\sigma_{a=s}^{\text {left }} f(s, b) .
$$

TheOREM 1 [5, Theorems 3.2, 4.2, 4.3]. If $a \in A^{n}, b \in A^{m}$ and $f \in \operatorname{Poly}_{n+m}^{p}$, then with no restriction there is inclusion

$$
f \sigma^{\text {left }}(a, b) \subseteq \sigma^{\text {left }} f(a, b) .
$$

If $a \in A^{n}$ is commutative and commutes with $b \in A^{m}$ then there is equality

$$
\sigma^{\text {left }} f(a, b)=\sigma_{a=a}^{\text {left }} f(a, b) .
$$

If the whole system $(a, b) \in A^{n+m}$ is commutative then there is equality

$$
\sigma^{\text {left }} f(a, b)=f \sigma^{\text {left }}(a, b) .
$$

These results are valid [7, Theorems 4.2, 4.3] if we replace each commutivity condition by the corresponding "quasi-commutivity" requirement [7, Definition 3.1].

3. Tensor products. If $A$ and $B$ are complex Banach algebras then we shall denote by $A \otimes B$ the completion of the algebraic "tensor product" $A \otimes_{C} B$ with respect to some uniform crossnorm [3], [11] which is compatible with the multiplication $(a \otimes b)\left(a^{\prime} \otimes b^{\prime}\right)=\left(a a^{\prime}\right) \otimes\left(b b^{\prime}\right)$. Thus elements of the form $\sum_{r=1}^{R} a_{r} \otimes b_{r}$ form a dense subspace, elements of the form $a_{1} \otimes b_{1}$ have norm $\left\|a_{1}\right\|\left\|b_{1}\right\|$, and for every pair of bounded linear functionals $\varphi \in A^{*}$ and $\psi \in B^{*}$, the linear functional

$$
\varphi \otimes \psi: \sum_{r=1}^{R} a_{r} \otimes b_{r} \rightarrow \sum_{r=1}^{R} \varphi\left(a_{r}\right) \psi\left(b_{r}\right)
$$


is bounded, and extends to the product $A \otimes B$.

THEOREM 2. If $a \in A^{n}$ and $b \in B^{m}$ are arbitrary then the system

$(a \otimes 1,1 \otimes b)=\left(a_{1} \otimes 1, a_{2} \otimes 1, \ldots, a_{n} \otimes 1,1 \otimes b_{1}, \ldots, 1 \otimes b_{m}\right)$

has left spectrum given by the product

$$
\sigma_{A \otimes B}^{\text {left }}(a \otimes 1,1 \otimes b)=\sigma_{A}^{\text {left }}(a) \times \sigma_{B}^{\text {left }}(b) .
$$

Similarly for the right spectrum; for single elements $a=a_{1} \in A$ and $b=b_{1} \in B$ there is inclusion

$$
\partial\left(\sigma_{A}(a) \times \sigma_{B}(b)\right) \subseteq \sigma_{A \otimes B}^{\mathrm{joint}}(a \otimes 1,1 \otimes b) \subseteq \sigma_{A}(a) \times \sigma_{B}(b) .
$$

Proof. The left-hand side of (3.2) is obviously included in the right; if, conversely, $s \in C^{n}$ is in $\sigma_{A}^{\text {left }}(a)$ and $t \in C^{m}$ in $\sigma_{B}^{\text {left }}(b)$, then the systems $a-s$ and $b-t$ generate proper closed left ideals $M$ and $N$ in $A$ and $B$. By the Hahn-Banach theorem there exist bounded linear functionals $\varphi \in A^{*}$ and $\psi \in B^{*}$ for which $\varphi(1)=\psi(1)=1$, while $\varphi(M)=\psi(N)=\{0\}$. Now the functional $\varphi \otimes \psi$ of (3.1) annihilates the left ideal generated by the system $((a-s) \otimes 1,1 \otimes(b-t))$ in the algebra $A \otimes B$, but not the identity $1 \otimes 1$. This puts $(s, t) \in C^{n+m}$ in the left spectrum of the system $(a \otimes 1,1 \otimes b)$.

For the inclusion (3.3) we use the fact [5, Lemma 4.1] that the topological boundary of the spectrum of a single element in a Banach algebra lies in the intersection of its left and right spectra.

4. Spectral mapping theorems. The combination of (3.2) from Theorem 2 with (2.10) from Theorem 1 gives at once

THEOREM 3. If $a \in A^{n}$ and $b \in B^{m}$ are commuting systems of elements, and $f \in$ Poly $_{m+n}^{p}$, then there is an equality

$$
\sigma_{A \otimes B}^{\text {left }} f(a \otimes 1,1 \otimes b)=f\left(\sigma_{A}^{\text {left }}(a) \times \sigma_{B}^{\text {left }}(b)\right) .
$$

Similarly for right spectra; for single elements $a=a_{1} \in A$ and $b=b_{1} \in B$, and one polynomial in two variables $f=f_{1} \in \mathrm{Poly}_{2}$, there is equality

$$
\sigma_{A \otimes B} f(a \otimes 1,1 \otimes b)=f\left(\sigma_{A}(a) \times \sigma_{B}(b)\right) .
$$

Proof. For the second part apply (3.3), together with a simple observation about polynomials in two complex variables:

$$
f\left(\partial\left(\sigma_{A}(a) \times \sigma_{B}(b)\right)\right)=f\left(\sigma_{A}(a) \times \sigma_{B}(b)\right) .
$$

One way to see this is to count the zeroes of the polynomial $f(\cdot, w)-r$ in the interior of the compact set $\sigma_{A}(a)$, for each complex number $r$ and each point $w$ of $\sigma_{B}(b)$; compare Lemma 2.2 of [12].

The Brown-Pearcy result $[\mathbf{1}]$ is the case $f(a \otimes 1,1 \otimes b)=a \otimes b$, with 
$A=B=\mathscr{L}(E, E)$ for a Hilbert space $E$. Our arguments readily extend to Schechter's generalization [12], which covers the product of $n$ copies of $A=\mathscr{L}(E, E)$ for a Banach space $E$, and rational functions $f$ with no singularities on the joint spectrum. Note carefully the difference between the "joint spectrum" of Schechter's paper [12] and ours in (2.1).

If only one of the systems $a \in A^{n}$ and $b \in B^{m}$ is commutative we still, using (2.7) and (2.9) instead of (2.10), obtain a result sufficient to determine the spectrum of an "operator matrix":

THEOREM 4. If $a \in A^{n}$ is a commuting system, if $b \in B^{m}$ is arbitrary, and if $f \in$ Poly $_{m+n}^{p}$ is a system of polynomials, then there is equality

$$
\sigma_{A \otimes B}^{\text {left }} f(a \otimes 1,1 \otimes b)=\bigcup\left\{\sigma_{B}^{\text {left }} f(s, b): s \in \sigma_{A}^{\text {left }}(a)\right\} .
$$

Proof. The right-hand side of (4.4) is included in the left because, if $s \in C^{n}$ is in $\sigma_{A}^{\text {left }}(a)$ and $r \in C^{p}$ in $\sigma_{B}^{\text {left }} f(s, b)$, then by (3.2), the system $(s, r) \in C^{n+p}$ is in $\sigma_{A \otimes B}^{\text {left }}(a \otimes 1,1 \otimes f(s, b))$, and by (2.7), also in $\sigma_{A \otimes B}^{\text {left }}(a \otimes 1$, $f(a \otimes 1,1 \otimes b))$. Conversely if $r$ is in the left-hand side of (4.4) we apply (2.9) to find $s \in C^{n}$ for which $(s, r)$ is in $\sigma_{A \otimes B}^{\text {left }}(a \otimes 1, f(a \otimes 1,1 \otimes b))$, and use (2.7) again.

For the application to "operator matrices" take $B=C_{q q}$ to be the algebra of $q \times q$ complex matrices, so that the tensor product $A \otimes_{C} B$ is " $q \times q$ matrices with entries in $A$ ": All the uniform crossnorms give the same Cartesian product topology. If we take $b=\left(b_{11}, b_{12}, \ldots, b_{q q}\right) \in B^{q^{2}}$ to be the usual basis for the vector space $B$ then an arbitrary matrix can be written

$$
f(a \otimes 1,1 \otimes b)=\sum_{j, k=1}^{q} a_{j k} \otimes b_{j k} ;
$$

we claim that, for a commuting system of entries $a=\left(a_{11}, a_{12}, \ldots, a_{q q}\right)$,

$$
\sigma_{A \otimes B} f(a \otimes 1,1 \otimes b)=\left\{r \in C: 0 \in \sigma_{A} \operatorname{det}(f(a \otimes 1,1 \otimes b)-r I)\right\} .
$$

The result can be obtained [9, Chapter 5] by extending the numerical determinant theory: here we use (4.4) on the left-hand side of (4.6), and apply (2.4) to the right-hand side.

5. Multiplication operators. Associated with a system $a \in A^{n}$ of Banach algebra elements are the systems $L_{a}$ and $R_{a}$ of multiplication operators, where, for each $j=1,2, \ldots, n$,

$$
L_{a_{j}}(x)=a_{j} x \quad(x \in A) \quad \text { and } \quad R_{a_{j}}(x)=x a_{j} \quad(x \in A) .
$$

Lumer and Rosenblum obtained the analogue of (4.2), with $L_{a}$ and $R_{b}$ in place of $a \otimes 1$ and $1 \otimes b$, in the case $A=\mathscr{L}(E, E)$ for a Banach space $E$. To summarize a derivation of this result we recall the left and right 
"approximate point spectrum" [5, Definition 1.3] of a system of Banach algebra elements :

$$
\tau_{A}^{\text {left }}(a)=\left\{s \in C^{n}: \inf _{\|x\| \geqq 1} \sum_{j=1}^{n}\left\|\left(a_{j}-s_{j}\right) x\right\|=0\right\}
$$

and

$$
\tau_{A}^{\mathrm{right}}(a)=\left\{s \in C^{n}: \inf _{\|x\| \geqq 1} \sum_{j=1}^{n}\left\|x\left(a_{j}-s_{j}\right)\right\|=0\right\} .
$$

Of course these are subsets of the left and right spectra (2.2) and (2.3); there is equality if $A=\mathscr{L}(E, E)$ is the bounded linear operators on a Hilbert space [5, Theorem 2.5], [2], and for a single element $a=a_{1}$ the topological boundary of the spectrum includes the intersection of (5.2) and (5.3) [5, Lemma 4.1]. The results of Lumer and Rosenblum [10] can be derived from

THEOREM 5. If $A=\mathscr{L}(E, E)$ for a Banach space $E$, and if $S \in A^{n}$ and $T \in A^{m}$ are systems of bounded linear operators, then there is inclusion

$$
\tau_{A}^{\text {left }}(S) \times \tau_{A}^{\text {right }}(T) \subseteq \sigma_{\mathscr{L}(A, A)}^{\text {left }}\left(L_{S}, R_{T}\right) \subseteq \sigma_{A}^{\text {left }}(S) \times \sigma_{A}^{\text {right }}(T)
$$

and

$$
\tau_{A}^{\mathrm{right}}(S) \times \tau_{A}^{\mathrm{left}}(T) \subseteq \sigma_{\mathscr{L}(A, A)}^{\mathrm{right}}\left(L_{S}, R_{T}\right) \subseteq \sigma_{A}^{\mathrm{right}}(S) \times \sigma_{A}^{\mathrm{left}}(T) .
$$

For single operators $S=S_{1}$ and $T=T_{1}$ there is inclusion

$$
\partial\left(\sigma_{A}(S) \times \sigma_{A}(T)\right) \subseteq \sigma_{\mathscr{L}(A, A)}^{\mathrm{joint}}\left(L_{S}, R_{T}\right) \subseteq \sigma_{A}(S) \times \sigma_{A}(T) .
$$

ProOF. The arguments for (5.4) and (5.5) are extracted from the proofs of Theorem 9 and Theorem 10 of Lumer and Rosenblum [10]; then (5.6) follows in the same way as (3.3).

For one polynomial $f=f_{1}$ in two variables, and for operators $S=S_{1}$ and $T=T_{1}$ on a Banach space it follows, analogous to (4.3), that

$$
\sigma_{\mathscr{L}(A, A)} f\left(L_{S}, R_{T}\right)=f\left(\sigma_{A}(S) \times \sigma_{A}(T)\right) .
$$

This of course is the result of Lumer and Rosenblum [10, Theorem 10]. Also for a Hilbert space $E$ we obtain equality throughout (5.4) and (5.5), and hence analogues for Theorems 3 and 4.

\section{REFERENCES}

1. A. Brown and C. Pearcy, Spectra of tensor products of operators, Proc. Amer. Math. Soc. 17 (1966), 162-169. MR 32 \#6218.

2. L. A. Coburn and M. Schechter, Joint spectra and interpolation of operators, J. Functional Analysis 2 (1968), 226-237. MR 37 \#3364.

3. J. Gil de Lamadrid, Uniform cross norms and tensor products of Banach algebras, Duke Math. J. 32 (1965), 359-368. MR 32 \#8125.

4. P. R. Halmos, A Hilbert space problem book, Van Nostrand, Princeton, N.J., 1967. MR 34 \# 8178. 
5. R. E. Harte, Spectral mapping theorems, Proc. Roy. Irish Acad. 72A (1972), 89-107. 6. - The spectral mapping theorem in several variables, Bull. Amer. Math. Soc. 78 (1972), 870-874.

7. The spectral mapping theorem for quasicommuting systems, Proc. Roy. Irish Acad. 73A (1973), 7-18.

8. - Tensor products, multiplication operators and the spectral mapping theorem, Proc. Roy. Irish Acad. (to appear).

9. K. Hoffman and R. Kunze, Linear algebra, Prentice-Hall Math. Ser., Prentice-Hall, Englewood Cliffs, N.J., 1961. MR 23 \# A3146.

10. G. Lumer and M. Rosenblum, Linear operator equations, Proc. Amer. Math. Soc. 10 (1959), 32-41. MR 21 \#2927.

11. R. Schatten, $A$ theory of cross-spaces, Ann. of Math. Studies, no. 26, Princeton Univ. Press, Princeton, N.J., 1950. MR 12, 186.

12. M. Schechter, On the spectra of operators on tensor products, J. Functional Analysis 4 (1970), 95-99.

Department of Mathematics, University College, Cork, Ireland 